\title{
Early Perioperative Outcomes After Surgery for Sleep Apnea: A Current Review of the Literature
}

\author{
Brian Rotenberg
}

Published online: 3 December 2013

(c) Springer Science + Business Media New York 2013

\begin{abstract}
Hospitals routinely admit most patients with obstructive sleep apnea (OSA) who undergo surgery for OSA to a high intensity care unit for overnight monitoring after surgery. This practice, mostly based on historical evidence, is designed to monitor respiratory outcomes after surgery in the event of adverse events taking place. However, this also creates inconvenience for patients, is very expensive to the health care system, and uses significant hospital resources. The goal of this systematic review of the literature was to assess what the current evidence is to support this practice and to begin to answer the question of which patients are benefitting from this approach.
\end{abstract}

Keywords Obstructive sleep apnea .

Uvulopalatopharyngoplasty · Systematic review ·

Perioperative outcomes

\section{Introduction}

It has been estimated that roughly one in four men and one in ten women in the US has obstructive sleep apnea (OSA) [1]. Besides the significant increase in risk OSA places on individuals, particularly with respect to cardiovascular health and overall mortality, there has been increasing recognition of how OSA potentially affects patients undergoing surgery.

B. Rotenberg

Department of Otolaryngology, Head and Neck Surgery,

Schulich School of Medicine and Dentistry, University of

Western Ontario, London, ON, Canada

B. Rotenberg ( $\square)$

St. Joseph's Healthcare Centre, 268 Grosvenor St., London, ON N6A 4V2, Canada

e-mail: brian.rotenberg@sjhc.london.on.ca
Given the aging population, resulting from both enhanced survival and the movement of baby boomers into their senior years, as well as increasing recognition of OSA as a health risk, the impact of OSA on perioperative care and costs is predicted to increase dramatically over the next few decades. A recent editorial from the New England Journal of Medicine termed this anticipated increase a 'perioperative sleep apnea epidemic' [2]. Despite the contemporary move toward more out-patient and same-day surgeries, designed to reduce health care costs within severely taxed health care systems, guidelines have been published by the American Society of Anesthesiologists Task Force on Perioperative Management of patients with OSA that essentially call for all surgery patients with OSA to be monitored overnight [3]. In a recent survey of Canadian Anaesthesiologists, $75 \%$ agreed with these guidelines [4]. Other health organizations, such as the Society for Ambulatory Anesthesia, support similar strategies [5••].

A survey was done to determine the practice patterns of OSA surgery in the USA between 2000 and 2006 [6॰]. For $0.2 \%$ of all patients with OSA undergoing surgical treatment, the majority of surgical procedures were performed in the outpatient setting [6•]. Moreover, many authors have questioned the validity of the ASA guidelines on the basis of the lack of empirical evidence supporting the statements [2, 7-10]. Finally the concept of mandatorily admitting all patients with OSA to the hospital stands in contrast to the current fiscal reality of healthcare across North America where practice patterns are being assessed for economic viability. It is more expensive to admit patients to the hospital than discharge them, and the question arises whether or not patients are benefiting from the ASA guidelines' recommendation of admission. It is possible that there are a large number of patients being unnecessarily admitted to the hospital, with associated patient inconvenience, hospital utilization, and cost implications. 
The objective of the current systematic literature review is to examine currently published studies in which data on outcomes in patients undergoing same-day OSA surgery are available, specifically to determine the incidence, timing, nature, and severity of adverse events in such patients. As a secondary objective, this study assesses a larger body of evidence on patients who have undergone OSA surgery in the inpatient or overnight care setting, examining the incidence, timing, nature, and severity of adverse events.

\section{Methods}

A systematic review of the literature was performed. The first step toward identifying studies in which perioperative outcomes were identified following surgery for the treatment of OSA was to do a MEDLINE search using the following single search terms that were decided upon a priori: sleep apnea (number of listed abstracts, $n=27,692)$; tonsillectomy ( $n=9,183$ ); uvulopalatoplasty ( $n=193)$; uvulopalatopharyngoplasty (UPPP) $(n=768)$; tongue reduction ( $n=1,221)$; radiofrequency ablation ( $n=13,368)$; lingual tonsillectomy $(n=250)$; tracheostomy $(n=10,159)$; desaturation $(n=7,346)$; intubation $(n=59,485)$; intensive care $(n=155,532)$; perioperative outcomes $\quad(n=8,634)$; perioperative monitoring ( $n=4,039$ ). For terms with $n>1,000$, only the first 200 abstracts were reviewed to identify any potentially appropriate references. When no suitable abstracts were identified, it was assumed that further searching would be grossly inefficient, and no further search using that single search term was performed. Abstracts were searched from 1990 to 2013 inclusive, in the English language. Older studies were felt to be too different from modern practice in terms of both surgical and anesthetic techniques, and were therefore not searched. Only studies relating to adults were included-purely pediatric studies, or studies that included a mixed adult and pediatric population, were excluded. A statistician was employed to help with this initial search.

The second step was to create combined search terms, based upon what was gleaned from step 1 . This led to the following search terms: sleep apnea surgery $(n=6,300)$; sleep apnea surgery complications $(n=2,959)$; perioperative outcomes after sleep apnea surgery $(n=22)$; sleep apnea day surgery $(n=313)$; outpatient sleep apnea surgery $(n=121)$; uvulopalatoplasty outcomes $(n=23)$; UPPP outcomes $(n=60)$; tongue reduction outcomes ( $n=60)$; uvulopalatoplasty complications $(n=79)$; UPPP complications $(n=291)$; tongue reduction complications $(n=228)$.

The next step involved selecting those abstracts that either presented information on perioperative outcomes or read in such a way that such data were expected to be included in the full paper. For such abstracts, the full papers were sought and reviewed. The fourth and final step was to review the reference lists of every paper identified thus far to identify additional papers that warranted more in depth perusal.

These four steps led to the review of a total of roughly 2,900 abstracts, leading to the further detailed review of 62 papers, ultimately leading to 19 papers with data on outcomes following same day surgical procedures for OSA, and 13 additional papers with data on immediate postoperative outcomes and complications in non same-day procedures. These papers are summarized in Tables 1 and 2 . Papers were excluded from review for one of the following reasons: because they contained no extractable, empirical data on immediate postoperative outcomes; patients with OSA either were not included (e.g., interventions for patients who snore but without OSA) or confirmed via polysomnography; data from patients with OSA were not able to be differentiated from those with other conditions; there was no denominator from which to estimate complications rates (e.g., surveys of surgeons to estimate the number of postoperative deaths they had observed in their practice). Studies in which an intervention was found to be ineffective relative to conservative management also were excluded [11].

\section{Results}

Perioperative Outcomes and Complications Following Same-Day Discharge After Surgery for OSA

In total, 18 papers with a combined 2,160 patients who underwent same-day surgery for OSA were identified (Table 1). Of these, six involved single or multi-stage procedures that included UPPP. Most patients had moderate to severe OSA, being at least an AHI of 15 or more, and were symptomatic. Of the remaining 12 papers, 4 involved radiofrequency tissue ablation, 7 laser-assisted UPPP and 1 palatal implants with UP flaps. Most of these final 12 papers involved patients with mild to moderate OSA. Several other papers were reviewed, especially for laserassisted uvulopalatoplasty (LAUP) procedures, but they primarily involved snorers rather than patients with OSA [12-23], or they had both groups of patients and made no attempt to distinguish them [13, 14, 24]. These papers were only analyzed for OSA-specific data, with snoring-specific data being excluded.

Across the studies the overall incidence of any adverse event was $5.3 \%$. There were no deaths or major catastrophic events. The re-admission rate to the hospital after discharge (for any reason) was $0.4 \%$. The adverse events that did occur were surgical complications and were not 


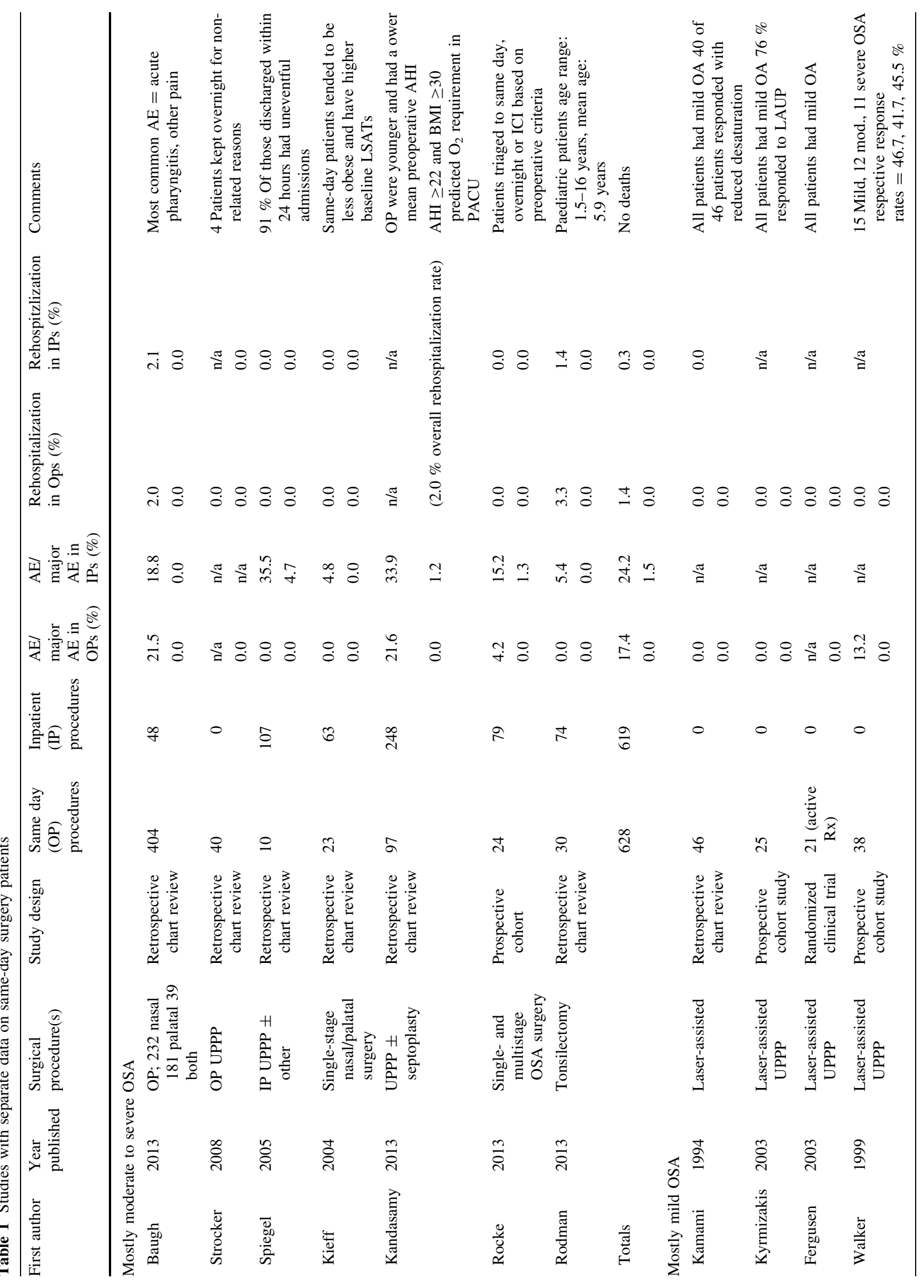




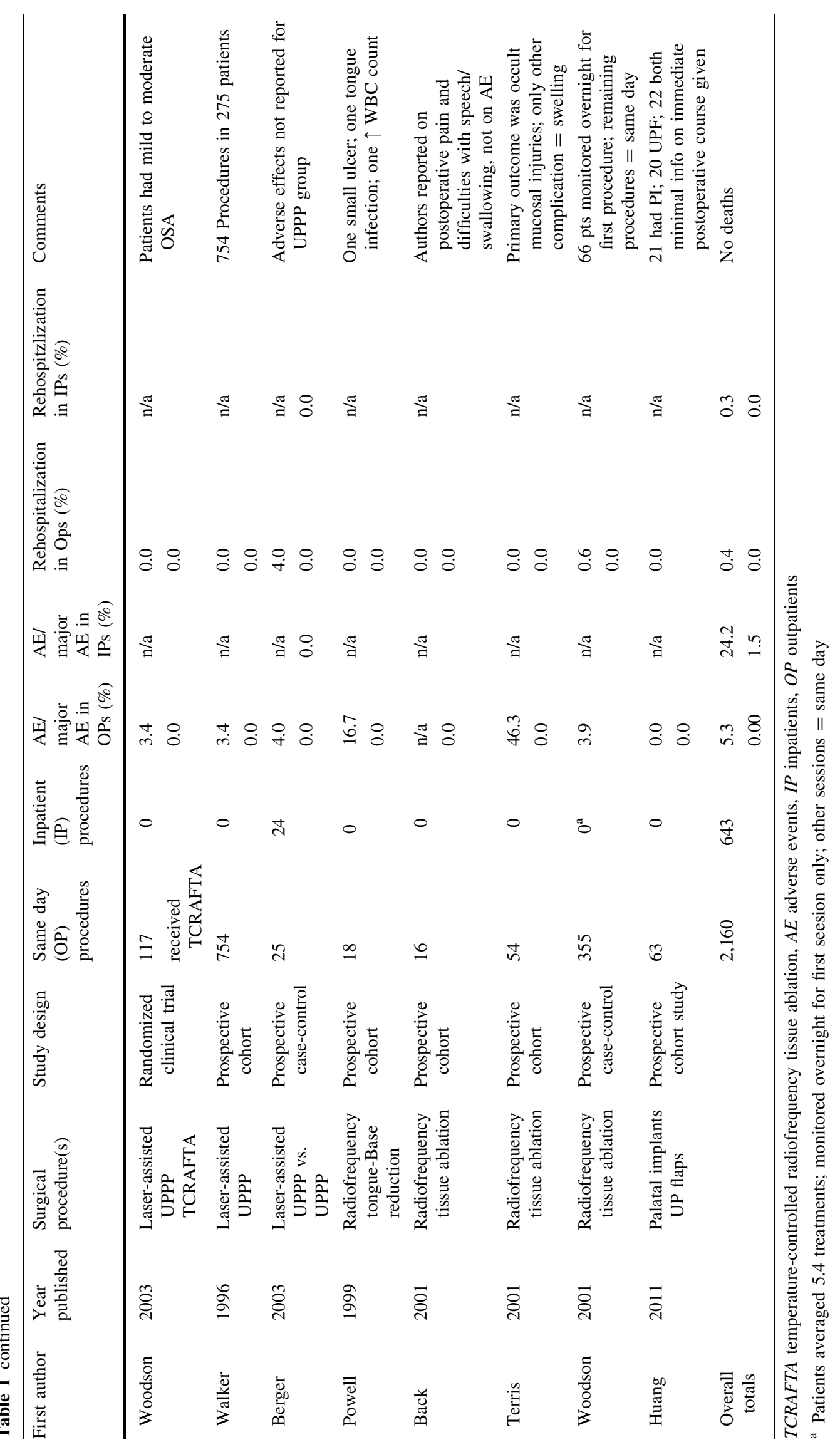




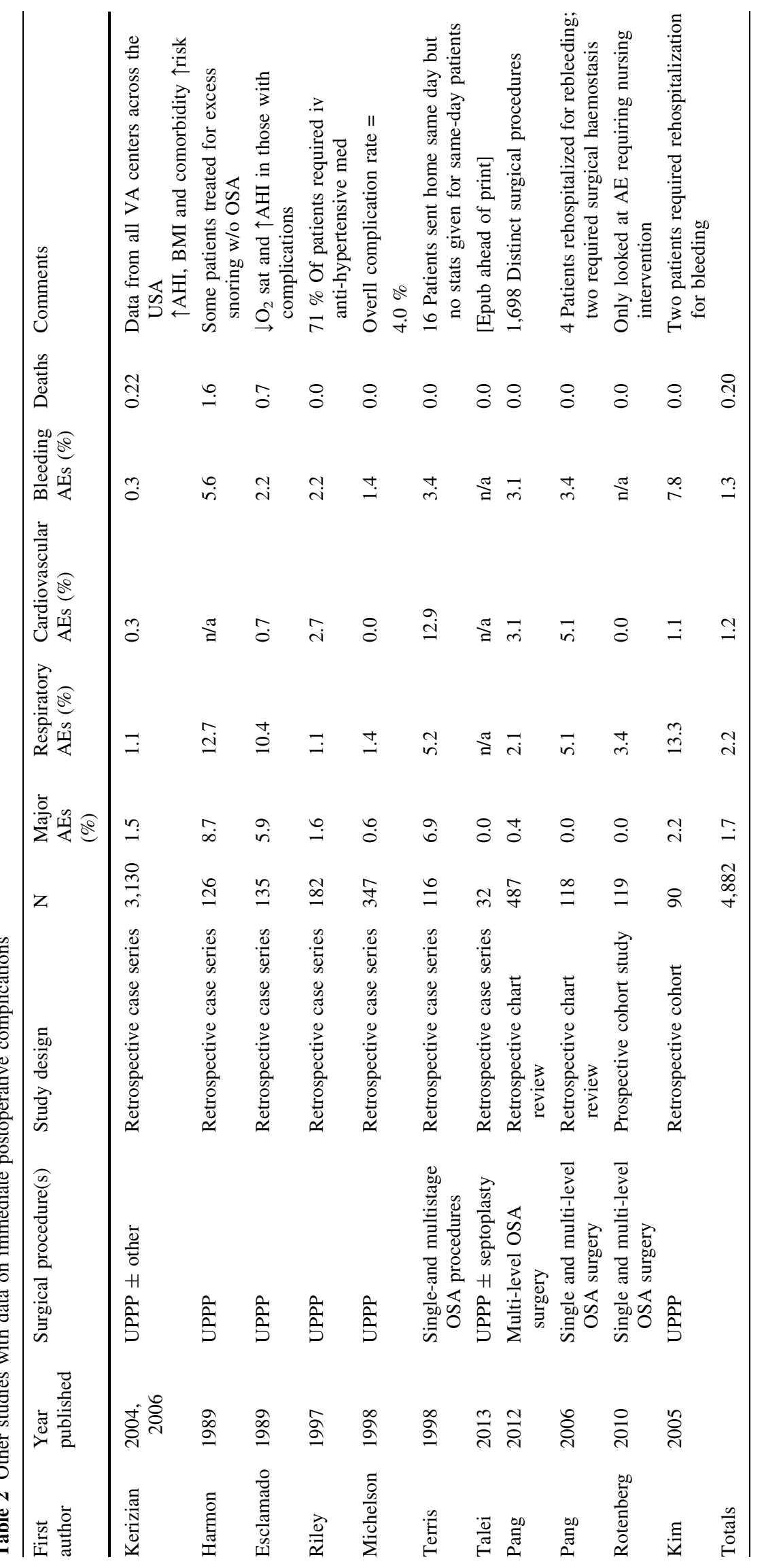


specifically related to the OSA. Corresponding percentages among patients undergoing conventional surgery, either single or multi-stage, were $17.4 \%$ rate of all adverse events, $0.0 \%$ death rate, and $1.4 \%$ rate of serious events. Meanwhile, among those undergoing laser-assisted procedures, done mainly as outpatient procedures, corresponding rates were in fact lower at 3.5, 0.0, and $0.1 \%$. Those undergoing radiofrequency tissue ablation had rates of 9.5 , 0.0 , and $0.5 \%$, respectively. Interestingly, among the 545 patients across these studies who were kept overnight or longer for unplanned reasons, rates for adverse events, major adverse events, re-hospitalizations, and death were $27.5,1.7,0.4$, and $0 \%$.

All six studies in which postoperative complications were assessed in patients undergoing standard (not laserassisted) UPPP and discharged home the same day were retrospective in design. In truth, in all but one study, some and usually the majority of patients were observed overnight or longer $[7,8,25,26 \bullet \cdot, 27]$. The exception, in which all surgeries were intended to be performed as same-day procedures, was a small retrospective chart review of 40 patients who underwent same-day UPPP [28]. In this study, four patients were kept overnight, though each instance was deemed to have been non-surgery related. These six studies had a heterogenous series of criteria to decide on same-day discharge versus admit; the choice was typically made on the basis of anatomical surgical site (if tongue base, then planned admission, otherwise potential discharge), on the basis of preoperative AHI severity (a wide range was cited in the literature), and on the issue of pain control and anticipated narcotic requirements. Certain comorbidities (such as known cardiovascular or respiratory disease) also resulted in planned admissions.

In few instances were adverse events compared statistically between the two groups. Given the non-random nature of group allocation, it would be expected that sicker patients and those who undergo a more complicated procedure would be more likely to be observed overnight, and more likely to experience an adverse event. There would be a clear bias toward more favorable outcomes, in general, in those discharged home early. On the other hand, more of those discharged same-day might be expected to return to the emergency room or to some other doctor with an unresolved issue.

The largest study for outpatient OSA procedures was a study of 452 patients, among whom 48 needed to be observed overnight, versus 404 sent home the same day [25••]. The primary objective of this study was to assess the safety of same-day OSA surgery, specifically looking at three safety indicators over the first 30 days post-surgery: emergency room visits, inpatient admission, and three or more visits to see a primary care physician. Catastrophic events that were recorded were myocardial infarction, deep venous thrombosis, stroke, pulmonary embolism, tracheostomy, transfusion, and death. In this large series, among the 404 same-day patients, there were 87 total adverse events, among which the most common were acute pharyngitis $(n=13)$, acute postoperative pain $(n=12)$, and bleeding $(n=5)$, without any OSA-specific events being recorded. There were no deaths and no catastrophic events. Twenty versus 17 percent of same-day patients returned for an ER visit within the 30-day follow-up period (NS), but the time to first ER visit was shorter in same-day patients (3 vs. 7 days, $p=0.03$ ). Otherwise, the two groups were similar [25••].

Retrospective attempts have been made to identify predictors of successful same-day discharge. In a study of 23 same-day patients versus $63 \mathrm{kept}$ at least overnight, Kieff et al. [8] found that the former tended to be less obese and to have higher baseline oxygen saturations. Kandasamy and Rotenberg found that 97 patients discharged home the same day were younger than the 248 kept overnight, and that a preoperative AHI $\geq 22$ and BMI $\geq 30$ both predicted desaturation in the post-anesthesia care unit (PACU). In both studies, it is possible that surgeons made decisions regarding same-day discharge related to possible risk factors such as age, obesity, or co-morbidites. In a novel twist, Rocke et al. [29•] developed a protocol of preoperative criteria to aid in the triage of patients to sameday surgery, over-night observation, or postoperative transfer to intensive care. The protocol consisted of three major criteria (multi-level surgery, a preoperative AHI $>60$, and a minimum oxygen desaturation $<75 \%$ from preoperative polysomnography), and two minor criteria (BMI $>35$ and significant comorbidities). Patients with one major or two minor criteria were allocated to the surgical intensive care unit (SICU), those with one minor criterion to the overnight observation unit, and those meeting no risk criteria were discharged home the same day. No serious complications occurred in those discharged home on the same day, versus $n=8$ and $n=5$ in those allocated to the SICU or the overnight observation unit. Moreover, all serious complications occurred within hours of surgery [29•]. Of great interest is that, using this preoperative triage scheme and based solely upon hospital room charges, the authors predicted annual savings of $\$ 125,000$ per year at their hospital [29•].

\section{Outcomes and Complications Following Hospital Admission After Surgery for OSA}

Table 2 lists studies relating to immediate postoperative complications as they pertain to OSA. Of the 13 studies in which immediate postoperative outcomes were assessed after OSA surgery, 10 were retrospective chart reviews [10, $30-34,35 \bullet \cdot, 36-38$ ], 1 a large prospective survey of records 
in a central database reported in 2 different papers [16, 40], 1 was prospective [41•], and 1 an unclear report of data collected by an agency in Sweden [39]. This final study by Carenfelt et al. [39] entailing the largest number of 'subjects' was reported in a one-page letter to the Lancet. No methodological details were provided other than a survey of 37 Swedish hospitals, with 35 positive responses. The denominator used (absolute number of UPPP and LAUP cases) seems to be a gross estimate, given the round numbers of 9,000 UPPP and 2,900 LAUP. Consequently, the numbers reported in this letter should be interpreted with caution. Another study by Haavisto et al. [31] was methodologically lacking in detail as well (Table 2). When including the Carenfelt et al. and Haavisto et al. data, which only exist for major adverse events and deaths, the incidence of serious adverse events was $1.8 \%$ and the mortality rate $0.09 \%$. Excluding these two studies, corresponding rates were 1.7 and $0.20 \%$. The incidences for respiratory and cardiovascular adverse events and significant bleeding were $2.2,1.1$, and $1.3 \%$, respectively.

By far the largest 'prospective' study on this topic was by Kezirian et al. [16, 40], which was reported across two different papers, in which the sample was 3,130 patients undergoing an inpatient UPPP procedure between 2000 and 2006 at any Veterans Affairs (VA) hospital in the US. The VA National Surgical Quality Improvement Program (NSQIP) prospectively collects 30-day complication and mortality data on any patients who undergo inpatient surgery at any VA hospital in the US. In this sample of 3,130 patients, $97 \%$ were male. Only seven deaths were recorded within 30 days postoperatively, for an overall incidence of $0.22 \%$, and none were thought to be related directly to the OSA. The rates for major adverse events, respiratory and cardiovascular adverse events, and significant bleeding in this sample were $1.5,1.1,0.3$, and $0.3 \%$, respectively. Two limitations of this study were (1) that data were collected generically, not specifically, to assess postoperative death and adverse events post-OSA surgery, so errors in procedure coding could have occurred; consequently, although data were collected prospectively, it was a retrospective decision to use them for the current study. (2) In addition, the sample was $97 \%$ male, which clearly limits its generalizability. A further problem that inherently arises when one study is so large relative to every other study analyzed is that this one study colors all the results. That being said, mortality rates ranged from zero to $1.0 \%$ for all other studies except that of Harmon et al., a small retrospective study of 126 patients published in 1989 [32]. Major adverse event rates varied more widely, but were only high in studies published in 1998 or earlier [30-32, 38]. The highest rate of major adverse events reported in the six studies since 1998 was $2.2 \%$ [33]. Some of this variation could be differences in the way in which major adverse events were defined or identified. For example, in the study by Rotenberg et al. [41•], no major OSA-specific respiratory adverse events were reported, but only events requiring a documented nursing intervention were counted.

In their review of studies assessing the effects and side effects of surgery for OSA and snoring, published in 2009, Franklin et al. identified a total of 37 deaths reported in the literature, though no incidence estimate could be generated because 22 of the deaths were reported in papers in which no denominator was provided (both were surveys of physicians or hospitals) [42, 43]. Our analysis was able to identify all the remaining 15 deaths, among which only 7 have been reported since 2000 , all in that single study of 3,130 patients treated at VA hospitals [16, 40]. Whether the decline from 30 deaths pre-2000 to 7 deaths from 2000 onward represents a true trend can only be speculated.

Three studies have attempted to identify risk factors for postoperative complications; however, as above, which complications were assessed varied between studies. Nonetheless, certain consistencies are noted. In all three studies [30, 31, 40], an elevated preoperative AHI predicted postoperative complications, especially respiratory, most notably oxygen de-saturation. Two studies identified low preoperative oxygen saturation as a predictor of postoperative de-saturation [30, 31], and two identified some measures of patients' weight (weight or BMI) [31, 40] as a risk factor for postoperative complications. Other preoperative comorbid conditions [40], and specifically cardiovascular comorbidity [31], also comprised a risk factor for postoperative complications. Of interest is that these four risk factors mirror the preoperative triage instrument proposed by Rocke et al. [29•], in which the three major criteria were multi-level surgery, a preoperative $\mathrm{AHI}>60$, and a minimum preoperative oxygen desaturation $<75 \%$, and the two minor criteria were BMI $>35$ and significant co-morbidities. However, one of these three studies must be considered methodologically weak [31].

Complications of surgical management of OSA can also present as medico-legal challenges for surgeons. In a very recent publication by Eloy's group [44], it was found that litigation associated with OSA more commonly revolves around perioperative issues than diagnostic ones. In reference to the topic of postoperative outcomes, pharyngeal surgeries were represented in over half of legal cases; $20.4 \%$ of cases were associated primarily with postoperative monitoring, and $7.4 \%$ of cases were associated with CPAP usage after surgery for OSA. Alarmingly, $73.3 \%$ of these cases were resolved with payment. Although this paper did not draw any direct conclusion between specifics of perioperative care 
and litigation outcomes, nor were case particulars discussed in detail, the authors did generally caution surgeons to be aware that these problems exist and stressed that (1) full informed consent discussion with patients should include perioperative issues and (2) airway management after surgery is a shared responsibility between the anesthesiologist and otolaryngologist. The best strategy is proactive planning.

\section{Conclusions}

From this exhaustive review of published scientific literature on the rates of immediate postoperative complications of OSA surgery, certain conclusions can be drawn:

(1) In patients undergoing surgery for OSA, the risk of severe adverse postoperative events is currently far lower than predicted by historical literature or contemporary perioperative guidelines, and is estimated at less than $2 \%$ by the findings of this systematic review. Additionally, no postoperative deaths have been reported in any OSA patient who has undergone same-day OSA surgery. Respiratory-related event rates in the modern literature are less than $1.5 \%$, and most were non-significant relating only to oxygen desaturations (which can only debatably be classified as a complication in the setting of OSA). If desaturations are ungrouped as a complication from other postoperative respiratory events, the adverse event rate drops far lower.

(2) Gaps still remain in our understanding of the risks and benefits of same-day versus inpatient OSA surgery, warranting prospective, inferential/quantitative research. However, based on the information in this review, health care centers should consider reviewing the admission protocols and consider sending patients undergoing OSA surgery home on the same day. Certain patients should be considered for admission, in particular those undergoing tongue base surgery, those with a higher preoperative apnea/hypopnea index, or those with high narcotic requirements. The policy of routinely admitting all patients with OSA to the hospital after surgery is excessive and not supported by modern evidence.

(3) The recommendations of ASA guidelines were based on the concept of monitoring for safety reasons after surgery. The question remains as to whether patients actually benefit from this practice. This issue has significant health economic implications, and further research should be performed in this area.

\section{Compliance with Ethics Guidelines}

Conflict of Interest Brian Rotenberg declares that he has no conflict of interest.

Human and Animal Rights and Informed Consent This article does not contain any studies with human or animal subjects performed by any of the authors.

\section{References}

Papers of particular interest, published recently, have been highlighted as:

- Of importance

•- Of major importance

1. Young T, Peppard PE, Gottlieb DJ. Epidemiology of obstructive sleep apnea: a population health perspective. Am J Respir Crit Care Med. 2002;165:1217-39.

2. Memtsoudis SG, Besculides MC, Mazumdar M. A rude awakening - the perioperative sleep apnea epidemic. N Engl J Med. 2013;368:2352-3.

3. Gross JB, Bachenberg KL, Benumof JL, et al. Practice guidelines for the perioperative management of patients with obstructive sleep apnea: a report by the American Society of Anesthesiologists Task Force on Perioperative Management of patients with obstructive sleep apnea. Anesthesiology. 2006;104:1081-93.

4. Friedman Z, Chung F, Wong DT, Canadian Anesthesiologists' Society. Ambulatory surgery adult patient selection criteria-a survey of Canadian anesthesiologists. Can J Anaesth. 2004; 51:437-43.

5. • Joshi GP, Ankichetty SP, Gan TJ, Chung F. Society for ambulatory anesthesia consensus statement on preoperative selection of adult patients with obstructive sleep apnea scheduled for ambulatory surgery. Anesth Analg. 2013;115:1060-8. This consensus statement is the most up to date practice parameter available on the topic of ambulatory surgery for sleep apnea.

6. - Kezirian EJ, Maselli J, Vittinghoff E, Goldberg AN, Auerbach AD. Obstructive sleep apnea surgery practice patterns in the United States: 2000-2006. Otolaryngol Head Neck Surg. 2010;143:441-7. This is a seminal paper reviewing a large number of patients undergoing sleep surgery.

7. Spiegel JH, Raval TH. Overnight hospital stay is not always necessary after uvulopalatopharyngoplasty. Laryngoscope. 2005; 115:167-71.

8. Kieff DA, Busaba NY. Same-day discharge for selected patients undergoing combined nasal and palatal surgery for obstructive sleep apnea. Ann Otol Rhinol Laryngol. 2004;113:128-31.

9. Rotenberg B, Theriault J, Pang K. Is overnight monitoring required for adult patients undergoing surgery for obstructive sleep apnea? Laryngoscope. 2011;121:692-3.

10. Mickelson-S A, Hakim I. Is postoperative intensive care monitoring necessary after uvulopalatopharyngoplasty? Otolaryngol Head Neck Surg. 1998;119:352-6.

11. Brown DJ, Kerr P, Kryger M. Radiofrequency tissue reduction of the palate in patients with moderate sleep-disordered breathing. J Otolaryngol. 2001;30:193-8.

12. Boudewyns A, Van De Heyning P. Temperature-controlled radiofrequency tissue volume reduction of the soft palate (somnoplasty) in the treatment of habitual snoring: results of a European multicenter trial. Acta Otolaryngol. 2000;120:981-5. 
13. Coleman SC, Smith TL. Midline radiofrequency tissue reduction of the palate for bothersome snoring and sleep-disordered breathing: A clinical trial. Otolaryngol Head Neck Surg. 2000;122:387-94.

14. Grontved AM, Karup P. Complaints and satisfaction after uvulopalatopharyngoplasty. Acta Otolaryngol Suppl. 2000;543:190-2.

15. Hukins CA. Arbitrary-pressure continuous positive airway pressure for obstructive sleep apnea syndrome. Am J Respir Crit Care Med. 2005;171:500-5.

16. Kezirian EJ, Weaver EM, Yueh B, et al. Incidence of serious complications after uvulopalatopharyngoplasty. Laryngoscope. 2004;114:450-3.

17. Larrossa F, Hernandez L, Morello A, et al. Laser-assisted uvulopalatoplasty for snoring: does it meet the expectations? Eur Respir J. 2004;24:66-70.

18. Rombaux P, Hamoir M, Bertrand B, et al. Postoperative pain and side effects after uvulopalatopharyngoplasty, laser-assisted uvulopalatoplasty, and radiofrequency tissue volume reduction in primary snoring. Laryngoscope. 2003;113:2169-73.

19. Sher AE, Flexon PB, Hillman D, et al. Temperature-controlled radiofrequency tissue volume reduction in the human soft palate. Otolaryngol Head Neck Surg. 2001;125:312-8.

20. Stuck BA, Sauter A, Hormann K, Verse T, Maurer JT. Radiofrequency surgery of the soft palate in the treatment of snoring. A placebo-controlled trial. Sleep. 2005;28:847-50.

21. Osman EZ, Osborne JE, Hill PD, Lee BW, Hammad Z. Uvulopalatopharyngoplasty versus laser assisted uvulopalatoplasty for the treatment of snoring: an objective randomised clinical trial. Clin Otolaryngol Allied Sci. 2000;25:305-10.

22. Said B, Strome M. Long-term results of radiofrequency volumetric tissue reduction of the palate for snoring. Ann Otol Rhinol Laryngol. 2003;112:276-9.

23. Pavelec V, Polenik P. Use of Er, Cr: YSGG versus standard lasers in laser assisted uvulopalatoplasty for treatment of snoring. Laryngoscope. 2006;116:1512-6.

24. Remacle M, Betsch C, Lawson G, Jamart J, Eloy P. A new technique for laser-assisted uvulopalatoplasty: decision-tree analysis and results. Laryngoscope. 1999;109:763-8.

25. • Baugh R, Burke B, Fink B, et al. Safety of outpatient surgery for obstructive sleep apnea. Otolaryngol Head Neck Surg. 2013;148:867-72. This is the most recent publication available reviewing safety data for outpatient surgery for sleep apnea.

26. • Kandasamy T, Wright ED, Fuller J, Rotenberg BW. The incidence of early post-operative complications following uvulopalatopharyngoplasty: identification of predictive risk factors. J Otolaryngol Head Neck Surg. 2013;42:15. Although a retrospective review, this series is the largest gathered to date of UPPP outcomes.

27. Rodman R, Boehnike M, Venkatasen N, Pine H. Discharge after tonsillectomy in pediatric sleep apnea patients. Int $\mathrm{J}$ Pediatr Otorhinolaryngol. 2013;77:682-5.

28. Strocker AM, Cohen AN, Wang MB. The safety of outpatient UPPP for obstructive sleep apnea: a retrospective review of 40 cases. Ear Nose Throat J. 2008;87:466-8.
29. • Rocke D, Sharp S, Wiener D, Puscas L, Lee WT. Effectiveness of a postoperative disposition protocol for sleep apnea surgery. Am J Otolaryngol. 2013;34:273-7. This study demonstrates early efforts at developing an outpatient protocol to assess monitoring disposition after surgery for sleep apnea.

30. Esclamado RM, Glenn MG, McCulloch TM, Cummings CW. Perioperative complications and risk factors in the surgical treatment of obstructive sleep apnea syndrome. Laryngoscope. 1989;99:1125-9.

31. Haavisto L, Suonpaa J. Complications of uvulopalatopharyngoplasty. Clin Otolaryngol Allied Sci. 1994;19:243-7.

32. Harmon JD, Morgan W, Chaudhary B. Sleep apnea: morbidity and mortality of surgical treatment. South Med J. 1989;82:161-4.

33. Kim JA, Lee J, Jung HH. Predictive factors of immediate postoperative complications after uvulopalatopharyngoplasty. Laryngoscope. 2005;115:1837-40.

34. Pang KP. Identifying patients who need close monitoring during and after upper airway surgery for obstructive sleep apnoea. J Laryngol Otol. 2006;120:655-60.

35. • Pang KP, Siow JK, Tseng P. Safety of multilevel surgery in obstructive sleep apnea: a review of 487 cases. Arch Otolaryngol Head Neck Surg. 2012;138:353-7. This series is the largest yet published assessing safety outcomes for multi-level sleep surgery.

36. Riley RW, Powell NB, Guilleminault C, et al. Obstructive sleep apnea surgery: risk management and complications. Otolaryngol Head Neck Surg. 1997;117:648-52.

37. Talei B, Cossu AL, Slepian R, Kacker A. Immediate complications related to anesthesia in patients undergoing uvulopalatopharyngoplasty for obstructive sleep apnea. Laryngoscope. 2013;123(11):2892-5. doi:10.1002/lary.24026.

38. Terris DJ, Fincher EF, Hanasono MM, Fee WE Jr, Adachi K. Conservation of resources: indications for intensive care monitoring after upper airway surgery on patients with obstructive sleep apnea. Laryngoscope. 1998;108:784-8.

39. Carenfelt C, Haraldsson PO. Frequency of complications after uvulopalatopharyngoplasty. Lancet. 1993;341:437.

40. Kezirian EJ, Weaver EM, Yueh B, et al. Risk factors for serious complication after uvulopalatopharyngoplasty. Arch Otolaryngol Head Neck Surg. 2006;132:1091-8.

41. - Rotenberg BW, Hu A, Fuller J, et al. The early postoperative course of surgical sleep apnea patients. Laryngoscope. 2010;120:1063-8. This study is unique in that it shows data on monitoring results of patients for a full $24 \mathrm{~h}$ after surgery for sleep apnea.

42. Fairbanks DN. Uvulopalatopharyngoplasty complications and avoidance strategies. Otolaryngol Head Neck Surg. 1990;102: 239-45.

43. Lee WC, Skinner DW, Prichard AL. Complications of palatoplasty for snoring or sleep apnoea. J Laryngol Otol. 1997;111: 1151-4.

44. Svider PF, Pashkova MD, Folbe MD, et al. Obstructive sleep apnea: strategies for minimizing liability and enhancing patient safety. Otolaryngol Head Neck Surg, 2103. doi:10.1177/ 0194599813504074. 